\title{
Case Report: Management of an Early Third Trimester Large Adnexal Mass in a Singleton Pregnancy
}

\author{
Lindsay Rucker*, MD, Zachary Walker, MD and Brian Casey, MD \\ Department of Obstetrics \& Gynecology, University of Alabama at Birmingham, Alabama, USA
}

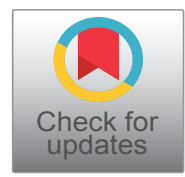

*Corresponding author: Lindsay Rucker, MD, Department of Obstetrics \& Gynecology, University of Alabama at Birmingham, $17006^{\text {th }}$ Avenue S., Birmingham, AL 35233, USA, Tel: (205)-934-5631, Fax: (205)-975-6411

\begin{abstract}
Background: Adnexal masses are increasingly being diagnosed in pregnancy. They are typically either managed expectantly or surgically in the early second trimester; however, limited reports on interventions for management in the third trimester.

Case: A 34-year-old G2P1001 patient had a right ovarian cyst found at $82 / 7$ measuring $10.35 \times 10.2 \times 8.38 \mathrm{~cm}$. It increased to $15 \times 11 \times 15 \mathrm{~cm}$ at $301 / 7$ and became symptomatic. She underwent percutaneous drainage (PCD) at $302 / 7$ with a decrease in size to $8.8 \times 4.2 \times 6.2 \mathrm{~cm}$ and symptom resolution. She was induced at $380 / 7$ for gestational hypertension and successfully delivered vaginally.

Conclusion: PCD of a simple cyst is a treatment option for women with significant symptoms in the third trimester.
\end{abstract}

\section{Introduction}

The incidence of adnexal masses diagnosed in pregnancy is on the rise given the routine use of ultrasound for pregnancy surveillance [1]. Currently 0.05 to $3.2 \%$ of pregnancies that result in a live birth are complicated by the diagnosis of an adnexal mass. Approximately 51 to $92 \%$ of adnexal masses identified during pregnancy resolve spontaneously. Ultrasound findings of adnexal masses that are greater than 5 centimeters in size or with complex morphology (e.g., multiloculated, septations, excresenses, or papillary projections) have been identified as indicators that a mass may persist throughout the duration of the pregnancy $[2,3]$. There are a variety of pathologic diagnoses for adnexal masses identified in pregnancy with varying incidence: corpus luteum cyst persisting into the second trimester (13-
$17 \%)$, benign cystic teratoma (7-37\%), serous cystadenoma (5-28\%), mucinous cystadenoma (3-24\%), endometrioma $(0.8-27 \%)$, paraovarian cysts $(<5 \%)$, and leiomyoma (1-2.5\%) [4].

The rate of ovarian malignancy in pregnancy is low and diagnosis relies heavily on imaging characteristics since traditional tumor markers are of less utility [1]. CA-125 levels are elevated in pregnancy and usually peak in the first trimester (7-251 units $/ \mathrm{mL}$ ) with subsequent decline in the second and third trimesters [2]. UItrasound findings that may indicate malignancy include decreased resistance in blood flow during Doppler examination, free abdominal/pelvic fluid, and those previously listed as indicative of mass persistence. If further morphological characterization is required, MRI is the next step [5].

Management of adnexal masses in pregnancy is founded on balancing the risks of intervention for the pregnancy and the risks of complications from a mass managed expectantly. Risks that may occur with observation include ovarian torsion, cyst rupture, bleeding, mechanical dystocia, and progression of an unidentified malignancy [3]. Alternatively, surgical intervention carries the risk of miscarriage, preterm labor, and thrombosis. Indications for surgery include suspicion of malignancy, size $>8-10 \mathrm{~cm}$, or an increased risk of ovarian torsion, cyst rupture, or obstruction of labor [6]. If surgical management is indicated, masses are typically excised via laparoscopy or laparotomy, ideally between 16 and 20 6/7 weeks gestation to minimize risks of spontaneous abortion in the first trimester and preterm

Citation: Rucker L, Walker Z, Casey B (2021) Case Report: Management of an Early Third Trimester Large Adnexal Mass in a Singleton Pregnancy. Obstet Gynecol Cases Rev 8:190. doi.org/10.23937/23779004/1410190

Accepted: January 27, 2021: Published: January 29, 2021

Copyright: (C) 2021 Rucker L, et al. This is an open-access article distributed under the terms of the Creative Commons Attribution License, which permits unrestricted use, distribution, and reproduction in any medium, provided the original author and source are credited. 


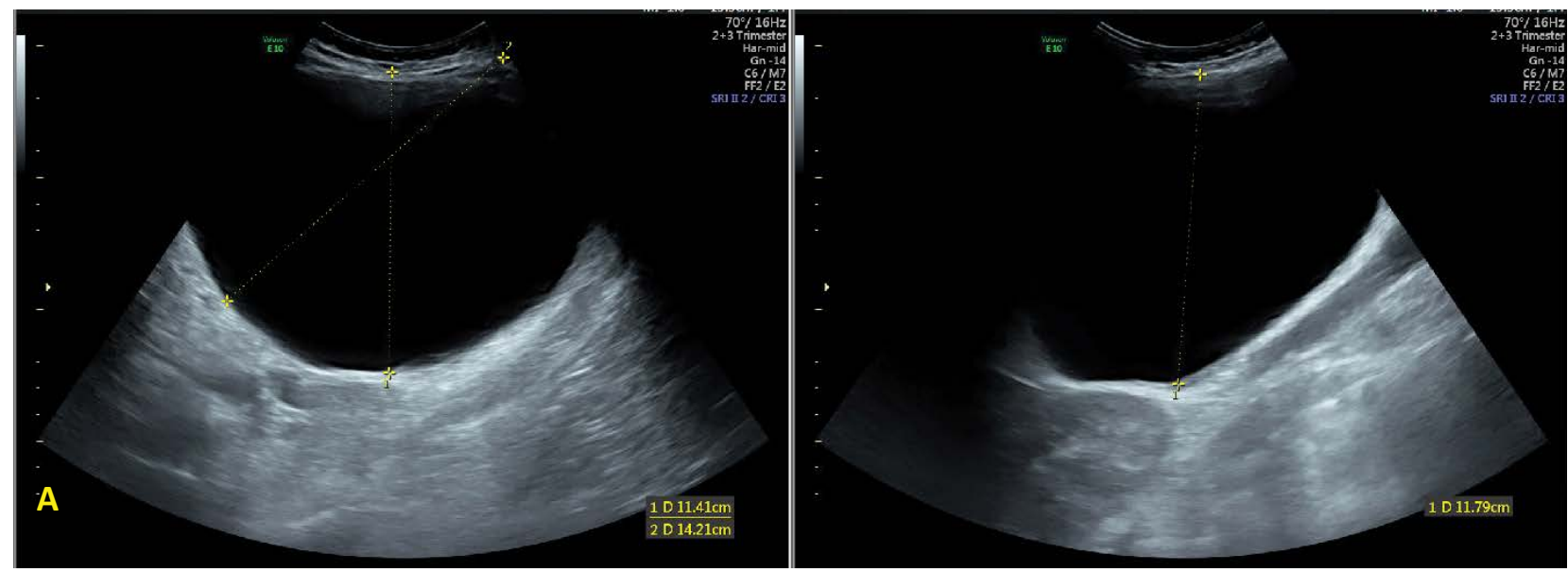

Figure 1: Ultrasound images at $301 / 7$ weeks demonstrating the simple cystic adnexal mass measuring $15 \times 11 \times 15 \mathrm{~cm}$.

labor in the third trimester [7]. Regardless of gestational age, torsion or hemodynamic instability secondary to cyst rupture are indications for surgery. Rarely, percutaneous and transvaginal drainage has been performed for simple, benign-appearing cysts. Little data exists on interventions performed for ovarian cysts in the third trimester [8].

\section{Case}

Patient is a 34-year-old G2P1001 had a simple cyst noted on the left ovary on her initial prenatal ultrasound at $82 / 7$ weeks measuring $10.35 \times 10.2 \times 8.38 \mathrm{~cm}$. The right ovary was unable to be visualized. An ultrasound at $163 / 7$ weeks for reassessment demonstrated acystic mass filling a large portion of the abdomen and pelvis, measuring $17 \times 9.7 \times 6.7 \mathrm{~cm}$. Tumor markers were obtained and were unremarkable with a CA-125 that was 21 units $/ \mathrm{mL}$ and CEA (carcinoembryonic antigen) that was $<1.8 \mathrm{ng} / \mathrm{mL}$. An abdominal MRI was performed at $166 / 7$ weeks that demonstrated a $12.9 \times 17.9 \times 7.3 \mathrm{~cm}$ T2 hyperintense, well-circumscribed cystic mass in the central abdomen without septations, tissue nodularity or wall thickening. After a comprehensive discussion between the patient and physician, surgery was deferred given the patient's lack of symptoms. However, by 18 weeks' gestation, the patient began to report worsening anorexia secondary to the mass.

Repeat surveillance imaging at 20 2/7 weeks' gestation revealed that the cyst had remained stable in size. Given her worsening symptoms, the patient was referred to a maternal-fetal medicine (MFM) specialist at $203 / 7$ for evaluation. At this time, continued expectant management was recommended as the patient was not demonstrating any signs of cyst rupture or ovarian torsion as bilateral Doppler flow was present in both ovaries. A plan was made for an ovarian cystectomy at time of a cesarean or a follow-up ultrasound 6 weeks postpartum if the patient delivered spontaneously.

The patient subsequently began to have worsening symptoms of anorexia, abdominal pain, and urinary in- continence. She was referred to our tertiary care center for further evaluation at $253 / 7$ weeks' gestation. Ultrasound at that time showed a simple $15.8 \times 13.5 \times 14.3$ $\mathrm{cm}$ left ovarian cyst. She was counseled that the cyst was likely benign and given that the optimal time for surgical management had passed, expectant management was again recommended. She was also referred to the Gynecologic Oncology Division where she was advised that, if necessary, cyst aspiration would be possible given the characteristics of the mass.

At 30 1/7 weeks' gestation a surveillance ultrasound showed a stable simple ovarian cyst measuring $15 \times 11$ $\times 15 \mathrm{~cm}$ (Figure 1 and Figure 2). However, the amniotic fluid index (AFI) was noted to be "subjectively low", though measured $7.69 \mathrm{~cm}$. This finding was consistent with a previous ultrasound scan from approximately two weeks prior by the patient's primary obstetrician that showed oligohydramnios with an AFI of $2.95 \mathrm{~cm}$. Given the concern for potential preterm rupture of membranes (PPROM), the patient was sent to the labor and delivery (L\&D) unit for further evaluation.

The patient was unable to provide a confirmatory history of PPROM because of difficulty with urinary incontinence for approximately 3 months prior to evaluation. Work up with sterile speculum evaluation was negative. However, the patient was found to be $3 \mathrm{~cm}$ dilated and $50 \%$ effaced on exam. The patient also complained of pelvic pain and anorexia. Given the escalation in her symptoms, the patient very strongly desired removal of the adnexal mass. She was admitted for concern for preterm labor (PTL) given cervical dilation in the setting of pelvic pain and further evaluation of potential treatment options for her adnexal mass. On admission, the patient's vital signs were normal and fetal testing was reassuring. The patient had left abdominal fullness accompanied by right uterine displacement. The patient was monitored on labor and delivery. She received a course of betamethasone for fetal lung maturity, 6 grams of magnesium for neuroprotection, and ampicillin for GBS prophylaxis. She was acontractile and 


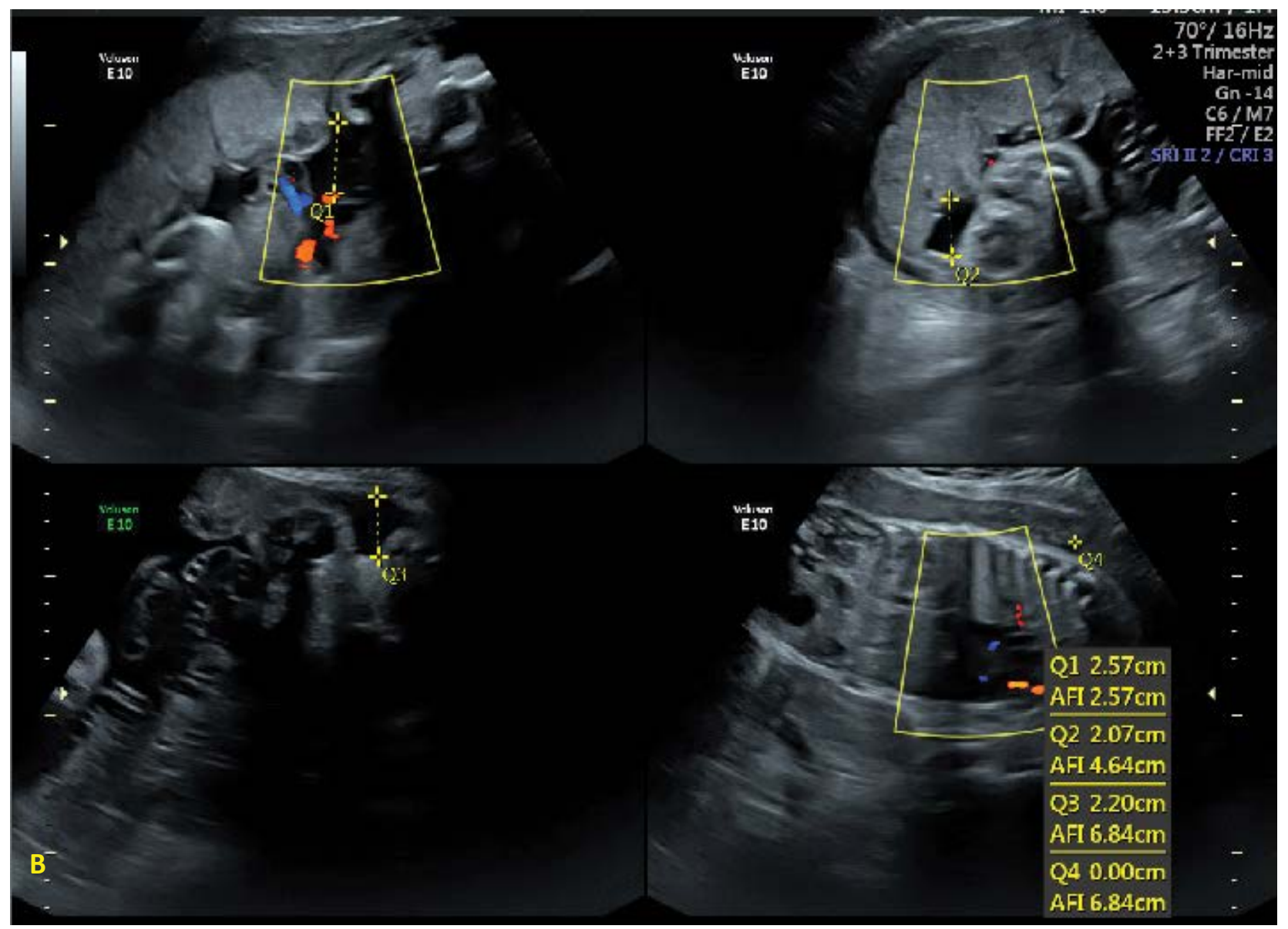

Figure 2: Ultrasound images at 30 1/7 weeks demonstrating subjectively low amniotic fluid.

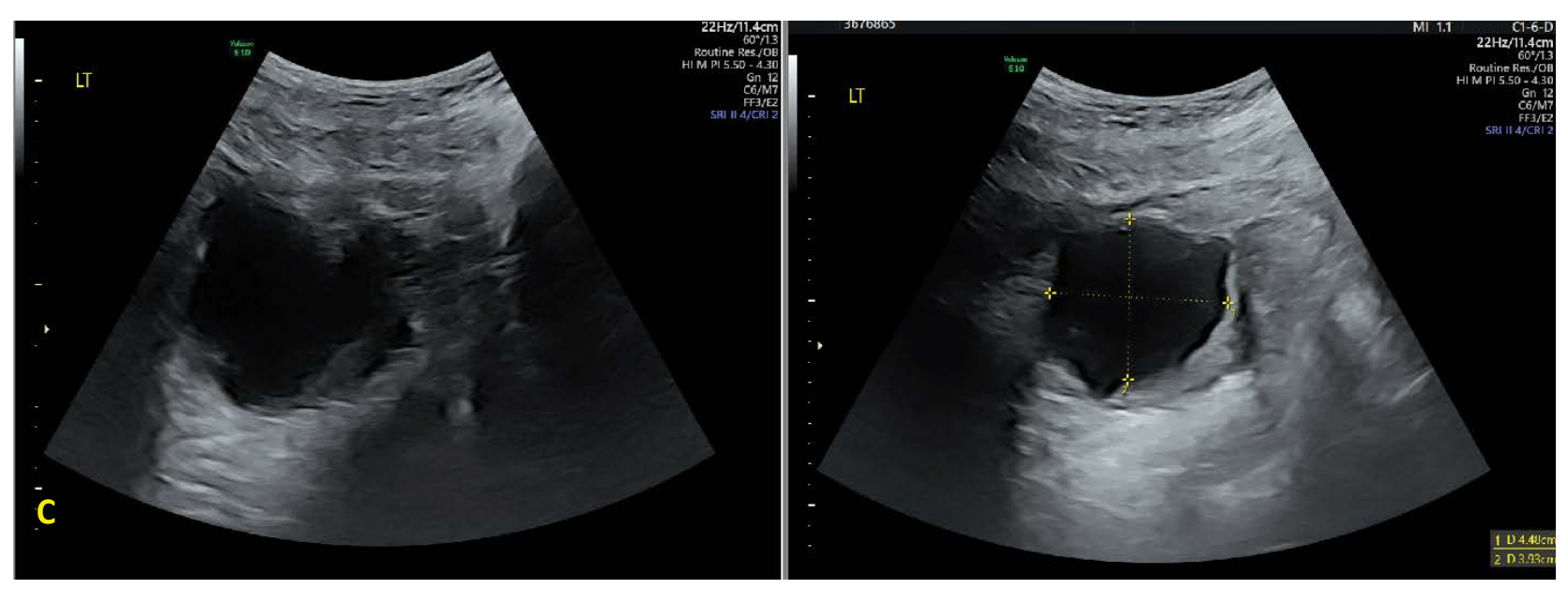

Figure 3: Ultrasound images at $373 / 7$ weeks demonstrating the decrease in size of the simple adnexal mass to $8.8 \times$ $4.2 \times 6.2 \mathrm{~cm}$ after aspiration of $950 \mathrm{~mL}$ of serous fluid.

her cervical exam remained unchanged after 12 hours of monitoring so she was therefore ruled out for PTL.

Gynecologic Oncology was consulted for recommendations regarding acute management options for the large adnexal mass. After a multidisciplinary discussion and consultation with the Radiology service for drainage of the cyst, the patient underwent ultrasound-guided percutaneous drainage (PCD) of the left ovarian cyst at $302 / 7$ weeks. $950 \mathrm{Ml}$ of serous fluid was removed and sent for cytologic evaluation. The procedure was performed under local anesthesia and the patient required one dose of intravenous pain medication postoperatively. On the first postoperative day, the patient noted significant improvement in her symptoms of pelvic pain, anorexia, and urinary incontinence and was discharged with a follow-up appointment in 3 weeks. The cytology report resulted as negative for malignancy. At her follow-up appointment at 34 2/7 weeks, the patient reported complete resolution of her previous symptoms. Repeat ultrasound at this time demonstrated a remaining $8.8 \times 4.2 \times 6.2 \mathrm{~cm}$ ovarian cyst and a nor- 
mal AFI of $17 \mathrm{~cm}$ (Figure 3). At $363 / 7$ weeks' gestation, the cyst remained unchanged at $8.3 \times 5.7 \times 2.3 \mathrm{~cm}$. At 37 $3 / 7$ weeks the patient was diagnosed with gestational hypertension for which she underwent labor induction at 38 weeks' gestation. She required manual placenta extraction, but otherwise had an uncomplicated delivery of a healthy female (Figure 4). She presented for her six week post-partum exam and remained asymptomatic. No further imaging or follow up appointments were scheduled by her primary provider.

\section{Conclusions}

Adnexal masses in pregnancy may either be managed expectantly or surgically via laparoscopy or laparotomy in the second trimester depending on size, symptoms, and morphological characteristics. Risks of expectant management of adnexal masses in pregnancy include ovarian torsion, cyst rupture, obstruction of labor, and delayed diagnosis of malignancy [3]. Risks of surgical intervention for adnexal masses include preterm labor, thrombosis, and fetal death [4]. PCD may be a less invasive alternative treatment option for persistent adnexal masses in pregnancy. Ultrasound assessment of mass size and complexity can be used to accurately predict malignancy. Identification of malignancy prior to PCD is imperative to avoid seeding the abdomen. A study by Thabet, et al. found that zero of nine patients with the fi- nal pathologic diagnosis of epithelial ovarian cancer had tumor seeding after drainage during a mean follow-up of 44 months [9]. The true risk of abdominal seeding after adnexal mass biopsy is largely unknown given the little existing data; however, the theoretical potential is important to consider in counseling patients in whom PCD is an option. Another risk of PCD is re-accumulation of fluid requiring retreatment which has been estimated to occur in $25 \%$ of non-pregnant patients [6]. Despite these risks, PCD of large cystic adnexal masses, especially simple cysts, in patients with significant symptoms should be considered.

This case demonstrates that, with thorough patient counseling and through shared decision making, PCD offers a viable treatment option for patients with rapidly enlarging adnexal masses and significant symptoms late in gestation. Additionally, in our case and a previous case of PCD of an adnexal mass during pregnancy by Bannon, et al., oligohydramnios initially noted on US resolved with drainage of the adnexal mass [7]. At this time, there has not been an association between oligohydramnios and adnexal masses documented in the literature.

\section{Authors Contribution}

All authors have contributed equally to the work.

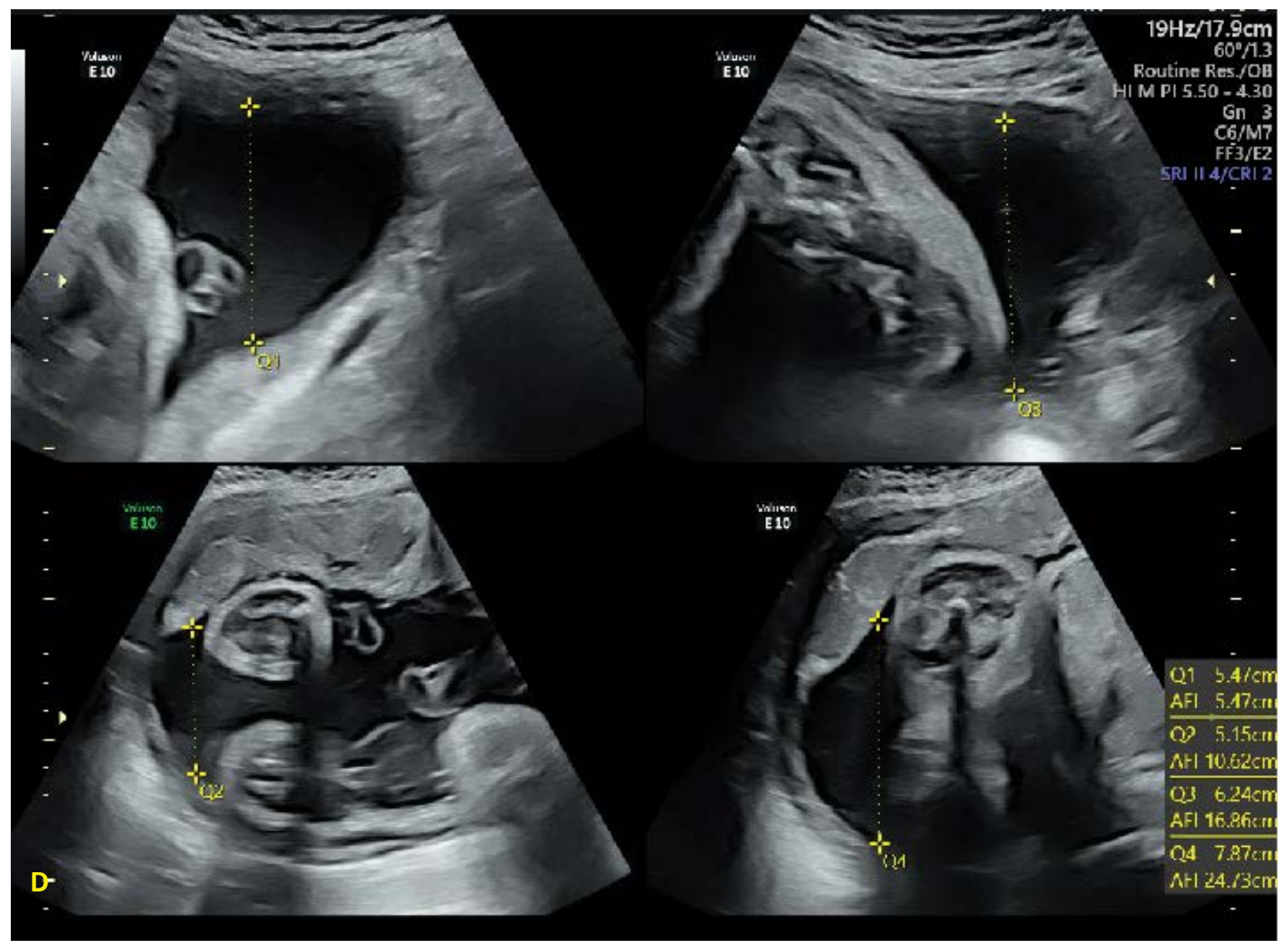

Figure 4: Ultrasound images at $373 / 7$ demonstrating increase in AFI to 24.73 after aspiration of the adnexal mass. 


\section{References}

1. Oprescu ND, Ionescu CA, Dragan I, Fetecau AC, Said-Moldoveanu AL, et al. (2018) Adnexal masses in pregnancy: Perinatal impact. Rom J Morphol Embryol 59: 153-158.

2. American College of Obstetricians and Gynecologists (2016) Practice Bulletin No. 174: Evaluation and management of adnexal masses. Washington.

3. Cavaco-Gomes J, Jorge Moreira C, Rocha A, Mota R, Paiva $V$, et al. (2016) Investigation and management of adnexal masses in pregnancy. Scientifica (Cairo).

4. Hoover K, Jenkins TR (2011) Evaluation and management of adnexal mass in pregnancy. Am J Obstet Gynecol 205: 97-102.
5. Hakoun AM, AbouAl-Shaar I, Zaza KJ, Abou-Al-Shaar H, Salloum MNA (2017) Adnexal masses in pregnancy: An updated review. Avicenna J Med 7: 153-157.

6. Leiserowitz GS (2006) Managing ovarian masses during pregnancy. Obstet Gynecol Surv 61: 463-470.

7. Bannon LC, Hayes KG, Porter KB (2015) Percutaneous drainage of a rapidly enlarging simple ovarian cyst in the third trimester. Mil Med 180: e1118-e1120.

8. Thabet A, Kalva SP, Liu B, Mueller PR, Lee SI (2012) Interventional radiology in pregnancy complications: Indications, technique, and methods for minimizing radiation exposure. Radiographics 32: 255-274.

9. Thabet A, Somarouthu B, Oliva E, Gervais DA, Hahn PF, et al. (2014) Image-guided ovarian mass biopsy: Efficacy and safety. J Vasc Interv Radiol 25: 1922-1927. 